\title{
Cranioplasty, Head Trauma and Neuropsychological Rehabilitation
}

\section{Espinosa-Gil Rosa María ${ }^{1 *}$, Raya-Güimil David ${ }^{2}$ and Monteagudo Santamaría María ${ }^{3}$}

${ }^{1}$ FEA Clinical Psychology, Rehabilitation Service, Hospital Clínico Universitario Virgen de la Arrixaca, Murcia, Spain

${ }^{2}$ Resident of Clinical Psychology, Multiprofessional Teaching Unit of Mental Health

of the Region of Murcia, Spain

${ }^{3}$ FEA Rehabilitation, Head of The Physical Medicine and Rehabilitation Service,

Rehabilitation Service, Hospital Clínico Universitario Virgen de la Arrixaca, Murcia,

Spain

*Corresponding Author: Espinosa-Gil Rosa María, FEA Clinical Psychology,

Rehabilitation Service, Hospital Clínico Universitario Virgen de la Arrixaca, Murcia,

Spain.
Received: July 01, 2021

Published: July 23, 2021

(C) All rights are reserved by Espinosa-Gil

Rosa María., et al.

\section{Abstract}

Decompressive craniectomy in people suffering from head injuries have been performed since the early twentieth century. At first they were performed in both hemispheres and today it is usually performed in the affected hemisphere. Although it is usually the last measure to be taken by neurosurgeons due to the strong risk it entails at the functional level, its practice is still necessary for some patients.

Sometimes patients have a comminute cerebral fracture so it is very difficult to re-implant the intact skull after the indicated period, when edematous symptoms are reduced and it is necessary to resort to other grafts.

This paper presents the results of two patients with TBI after performing a cranioplasty and subsequent incorporation into a neuropsychological rehabilitation program for one year (cognitive stimulation and interfamilial-intercultural groups) in a rehabilitation service. An improvement was observed in different neuropsychological parameters, being an indicator that cranioplasty improved cerebral blood flow and cortical atrophy. Likewise, the findings suggest that after 6 months, the brain organization has already occurred and no further improvements are obtained in the parameters and neuropsychological measures studies.

Keywords: TBI; Cranioplasty; Clinical Psychology; Neuropsychology; Neuropsychological Rehabilitation

\section{Abbreviations}

MCA: Middle Cerebral Artery; AVBD: Basic Activities of Daily Living; CD: Decompressive Craniectomy; EDTC: Transcranial Doppler Ultrasound; HTIC: Intracranial Hypertension; TBI: Head Trauma; RESCUEIcp: Randomized Evaluation of Surgery with Craniectomy for Uncontrollable Elevation of Intracranial Pressure 2016

\section{Introduction}

Neuropsychological alterations of acquired brain damage

Within the clinical neuropsychology, the different aspects that may be affected in acquired brain damage are studied with the aim of establishing an adequate treatment plan. The most frequently 
altered domains in these cases are memory, attentional processes, and executive functions.

The prefrontal cortex is the brain region where the most complex and evolved cognitive functions of the human being are located, and it is attributed an essential role in activities as important as creativity, the execution of complex activities, the development of formal operations of thought, social behavior, decision-making and ethical and moral judgment $[1,2]$. Executive functions are defined as a set of skills that are involved in the generation, supervision, regulation, execution and readjustment of appropriate behaviors to achieve complex objectives, especially those that are novel to the individual and that require a creative solution [3]. Sholberg and Mateer [4] consider executive functions to encompass a number of cognitive processes, including anticipation, choice of goals, planning, behavior selection, self-regulation, self-monitoring, and feedback.

The brain damage that occurs when there is a TCE is diffuse and does not only involve a focal injury. Hence, we are not only talking about an impairment of memory or an aphasic picture if it covers the temporal lobe, but also involved pathways of connection with other brain regions.

As for specific damage to the left hemisphere, Kolb and Wishaw [5] have identified eight symptoms associated with temporal lobe injuries: 1) alterations in sensation, perception and hearing; 2) alterations of the selective attention of the auditory and visual input; 3) alterations in visual perception; 4) alterations in the organization and classification of verbal material; 5) alteration in verbal comprehension; 6) long-term memory impairment; 7) personality changes, affective disorders and clinical paranoid onset; 8) altered sexual behavior.

The temporal lobes are involved in the primary organization of sensory input [6]. Individuals with temporal lobe lesions have difficulty naming and classifying by category. Left temporal lesions alter the knowledge of words and are associated with memory skills. Left temporal injuries have also been associated with memory alterations of non-verbal material, such as music and drawings. Damage to the temporal lobe can have dramatic effects on an individual's personality, with increased sexual behavior [7] or causing language disorders (aphasias) and inability to perceive objects (agnosias). Temporal lobe epilepsy has been associated with lan- guage disturbances (persevering speech), paranoia and aggressive behaviors [8].

As for damage to the right hemisphere, there is usually a predominance in alterations of spatial functions and attention [9].

What is a decompressive craniectomy and why are they performed?

It is a surgical procedure whose main medical indications are: a) intracranial hypertension secondary to ischemic or hemorrhagic stroke (mainly in malignant infarcts of the arteria cerebral media or less frequently, in ischemic lesions of the posterior fossa); b) post-traumatic intracranial hypertension (as a second-level measure when previous treatments for the control of intracranial pressure fail or in patients requiring urgent surgery, usually for evacuation of blood collection); c) uncontrollable cerebral edema after a scheduled craniectomy. Surgery is usually the last of the treatments for the control of post-traumatic refractory HTIC.

There are two prospective, randomized, multicenter, controlled studies on the benefits of decompressive craniectomy in posttraumatic brain injury. Cooper., et al. [10] conducted the DECRA study between 2002 and 2010 to test how it improves functional prognosis in patients with refractory intracranial hypertension following post-traumatic injury. The sample consisted of 155 patients from 15 hospitals in Australia, New Zealand and Saudi Arabia, aged between 15 and 59 years. Patients who maintained HF pressures greater than $20 \mathrm{mmHg}$ after 72 hours were randomly divided into two groups: a) hypothermia and/or barbiturate coma; b) surgical group: bifront parietotemporal decompressive craniectomy. The results were negative: although in the surgical group the HTIC and the stay in the ICU decreased, at 6 months the mortality rates were similar in both groups and the degree of severe disability was worse in the group of the patients operated on, so craniectomy was associated with a more unfavorable functional prognosis.

In the RESCUEIcp Trial [11], researchers included 400 patients from the Cambridge Department of Neurosurgery who had intracranial pressure above $25 \mathrm{mmHg}$ for 12 hours despite the application of first and second level measures. They were randomly divided into two groups: 1) usual medical treatment; 2) Surgical treatment, which consisted of unilateral front temporoparietal decompression. Lower mortality rates were observed in the surgically operated group but associated with higher levels of disability. 


\section{Cranioplasty and clinical implications}

Cranioplasty, or posterior cranial reconstruction is a technique dating back to the ancient Incas in Peru (2000 BC.C.), who used gold plates to camouflage a frontal defect as a result of trepanation [12]. It was from the work of Kocher [13] in the early twentieth century that the use of this surgical procedure spread. The goal of performing a cranioplasty is to protect the underlying brain, restore aesthetics, prevent brain atrophy, and reduce the risk of secondary seizures. The time elapsed between decompressive craniectomy and the decision to perform a cranioplasty is a controversial issue [14-16] due to the appearance of medical complications in this type of intervention.

Winkler., et al. [17] found in a study with 12 subjects an increase in the uptake of 18-fluoro-deoxy-glucose (18-FDG), a marker of cellular glycidic metabolism, of $12 \%$ and $4 \%$, in the sick and healthy hemispheres, respectively, after performing cranioplasty. They also found that the asymmetry between hemispheres in the uptake of 18-FDG decreased from 35 to $28 \%$ after surgery, and correlated it with their clinical findings: 10 of the 12 PET performed demonstrated an improvement in perfusion, and in all these cases a clinical improvement was observed. Table 1 shows other studies with similar findings.

\begin{tabular}{|l|c|c|c|}
\hline Authors & $\begin{array}{c}\text { Number of } \\
\text { Patients }\end{array}$ & $\begin{array}{c}\text { Image } \\
\text { mode }\end{array}$ & Conclusion \\
\hline $\begin{array}{l}\text { Suzuki., } \text { et } \\
\text { al. [18] }\end{array}$ & 6 & TCP & $\begin{array}{c}\text { Increase between } 2 \text { and } 20 \% \\
\text { on sick and healthy side } \\
\text { respectively }\end{array}$ \\
\hline $\begin{array}{l}\text { Won., } \text { et al. } \\
{[19]}\end{array}$ & 27 & TCP & $\begin{array}{c}\text { Increase from } 39.1 \text { to } 44.7 \\
\text { ml/100g/s and from } 42.9 \text { to } \\
47.2 \text { ml/100gr/s on the sick } \\
\text { and healthy sides respectively }\end{array}$ \\
\hline $\begin{array}{l}\text { Paredes., } \text { et } \\
\text { al. } \text { [20] }\end{array}$ & 49 & TCP & $\begin{array}{c}\text { Increase from } 101.86 \text { to } \\
117.17 \text { ML/100gr/s and from } \\
128.14 \text { to } 145.73 \text { ml/100gr/sg } \\
\text { on the sick and healthy sides } \\
\text { respectively }\end{array}$ \\
\hline
\end{tabular}

Table 1: Studies on cranioplasty and cerebral perfusion.

Erdogan., et al. [21] found in a study with 18 patients that all ipsilateral defect velocities measured by EDTC were significantly lower, while they were close to normal values on the healthy side. These low speeds increased to normal levels after plasty. Table 2 shows studies in this regard.

\begin{tabular}{|l|c|c|}
\hline \multicolumn{1}{|c|}{ Authors } & $\begin{array}{c}\text { Number of } \\
\text { patients }\end{array}$ & \multicolumn{1}{c|}{ Conclusion } \\
\hline Winkler., et al. [17] & 13 & $\begin{array}{c}\text { Increased velocity of Arteria } \\
\text { Cerebral Media after surgery }\end{array}$ \\
\hline Won., et al. [18] & 27 & $\begin{array}{c}\text { Slowing down in both Arteria } \\
\text { Cerebral Media and Arteria Ce- } \\
\text { rebral Izquierda after surgery }\end{array}$ \\
\hline $\begin{array}{l}\text { Paredes., } \text { et al. } \\
\text { [20] }\end{array}$ & 47 & $\begin{array}{c}\text { Increase Lindegaard index vari- } \\
\text { ation on the sick side of patients } \\
\text { who will improve. This variation } \\
\text { disappears after surgery. }\end{array}$ \\
\hline
\end{tabular}

Table 2: Studies on cranioplasty and transcranial Doppler ultrasound.

\section{Aim of the Study}

The present study aims to: a) demonstrate whether cranioplasty is associated with improvements in cognitive abilities in two patients after the implantation of heterologous plasty; and b) to check whether neuropsychological rehabilitation after cranioplasty improves cognitive functioning 6 months after surgery.

\section{Materials and Methods}

Participants

Patient 1 (left TCE)

A 38-year-old male who in October 2016 suffered a TCE in his left hemisphere (Figure 1). Craniotomy is performed for evacuation of acute hemorrhagic and subdural contusion. Replacement of bone flap per month, which had to be removed for E. coli infection. He presented bursting in the left temporal lobe with bleeding at multiple points towards the subdural space, left parietal and frontal hemorrhagic bruises. Decompressive craniectomy with Duragen-covered open dura mater. A year later, heterologous cranioplasty is performed.

\section{Patient 2 (right TCE)}

A 31-year-old male with no relevant history. TCE in the right hemisphere after motorcycle accident with acute subdural hematoma, subsequent right hemispheric hematoma, which required craniectomy, leaving residual osseous defect. Glasgow of 15, bradypsychia, left 4/5 hemiparesis. Cranial bone defect in sunken right convexity, without scar abnormalities. Heterologous cranioplasty one year after decompressive craniectomy (Figure 2 and 3). Reopening of right fronto-parieto-temporal incision. Gallia dissec- 


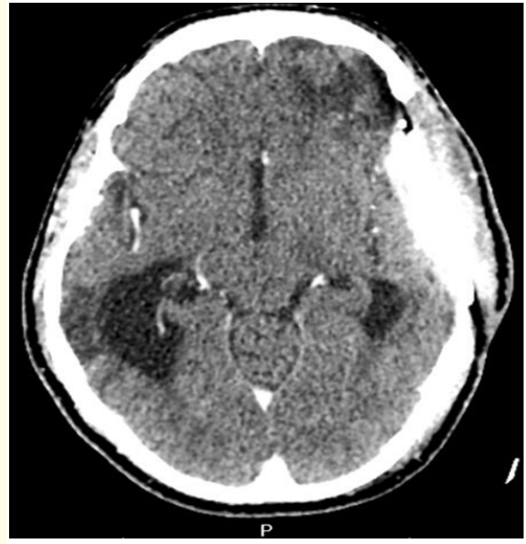

Figure 1: Patient with left TCE.

tion and temporary muscle flap. Placement of custom plasty and fixation with plates and screws. Temporary m. flap anchorage to osteosynthesis plates. Cranial CT showed right fronto-temporal encephalomalacica lesion and pale nucleus encephalomalácica attributable to a superficial and deep infarction in right MCA (Figure 4).

\section{Evaluation tools}

Clinical evaluation through initial interview and battery of neuropsychological tests composed of the following instruments:

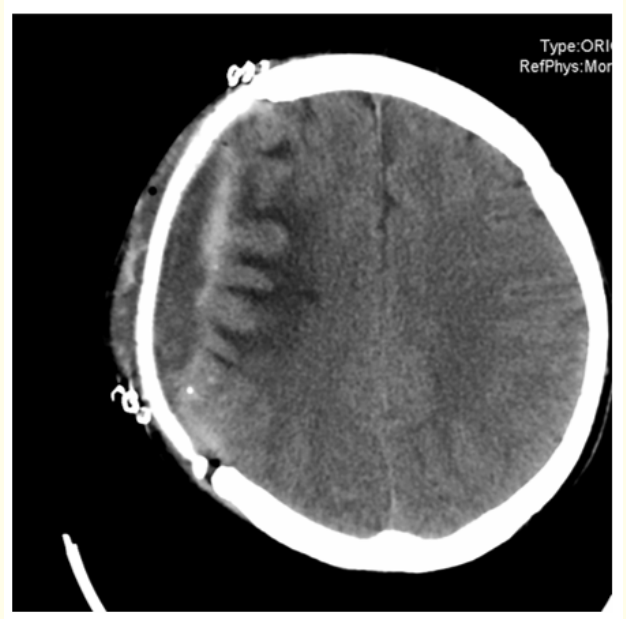

Figure 2: Patient with right TCE.
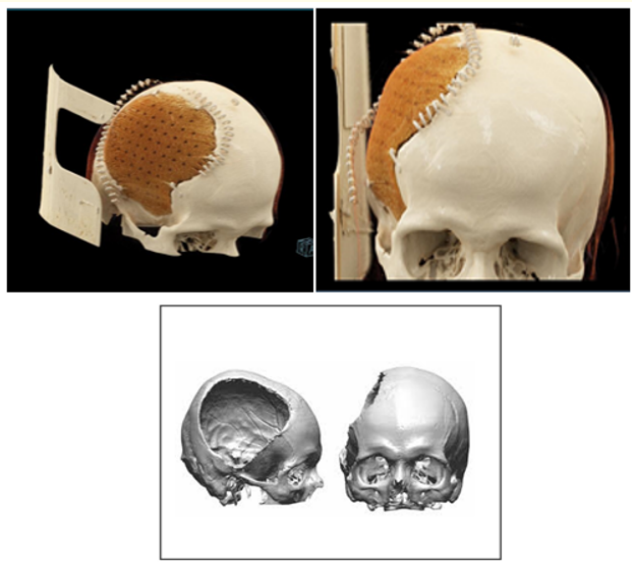

Figure 3: Cranioplasty on the right side.

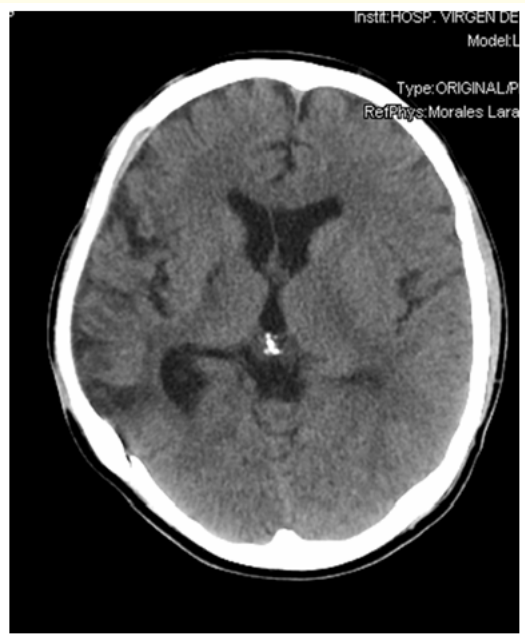

Figure 4: The right fronto-temporal encephalomalacica encephalomalacica.

Trail making test [22]

Neuropsychological test that evaluates different cognitive functions, including attention, psychomotor speed and cognitive flexibility. It consists of two parts: part A, in which it is intended to connect, by means of lines and consecutively, 25 numbers distributed randomly on a sheet; and part $\mathrm{B}$, in which the connection has to follow the same logic but joining numbers and letters alternately. 
WMS-III. Wechsler memory scale (Subtest Word List I and II) [23]

It is an individual application scale that evaluates different amnesic abilities. It can be applied between 16 years and 89 years and 11 months and has scales for spanish population. The word list subtest values immediate and deferred memory, pending learning and recognition of a list of words presented aurally.

\section{Mini mental state examination MMSE [24]}

It is a screening test widely used internationally to measure cognitive decline. It has a score scale ranging from 0-30 points in which scores below 26 are susceptible to possible cognitive decline.

\section{Barcelona-revised test [25]}

Neuropsychological scanning battery that assesses cognitive status. It includes a wide number of cognitive functions, such as language, orientation, attention, memory, reading, writing, praxis, recognition, memory and abstraction. The following tests were selected to evaluate memory: Naming by visual confrontation of sheets, objects and body parts; categorial evocation of animals and evocation of words beginning with $\mathrm{P}$.

\section{Complex king-osterrieth figure [26]}

It allows to evaluate the perceptual organization and also the visual memory in the people. It was originally used in individuals with brain injury, but has also subsequently been shown to be useful in the evaluation of cognitive functions in the non-lesional population. Consta of two phases: copy and memory playback.

\section{Frontal assessment battery FAB [27]}

Instrument that allows to evaluate the executive functions located in the frontal lobes quickly. It consists of 6 tests corresponding to each specific cognitive or behavioral domain related to the frontal lobes (conceptualization, mental flexibility, programming and motor planning, inhibitory control, sensitivity to interference and impulsivity. The maximum score is 18 and is obtained from the sum of each of its 6 tests, which are scored between 0 and 3 .

\section{Beck depression inventory, BDI-II [28]}

Spanish version. Scale of 21 items with response 0 to 3 points and score range of 0-63 that measures depressive symptomatology. Its internal consistency for psychiatric and non-psychiatric populations is $\alpha=.76$ and $\alpha=.95$ respectively.
Treatment

\section{Neuropsychological intervention models}

During the cycle of sessions that the 2 patients received, the attention intervention programs of Sohlberg and Matter, 2001 (working focused, sustained, selective, divided attention), declarative memory, the executive functions components of Sohlber and Matter were included. and the different executive functions of Professor Tirapu that has been supporting his research (Muñoz and Tirapu, 2004) (working memory proposed by Baddeley, cognitive flexibility, decision-making, process speed). They also completed naming and comprehension language tasks, along with abstraction tasks. All the activities were carried out with pencil and paper activities and some computer support to carry out the tasks that were most relevant in each of the patients. Within an approach where we do not forget social cognition, and the intervention from interfamily and intercultural groups that we carry out in the Rehabilitation service to address acquired brain damage (Espinosa and Monteagudo 2020). Systemic family therapy was also performed. We do not forget the application of strategies and self-instructions to carry out mainly attentional tasks.

We follow the principles of Sohlberg and Mateer for a rehabilitation program, which guide the selection process of tasks and intervention strategies:

- Use a treatment based on a theoretical model.

- Use hierarchically organized tasks.

- Give the opportunity to repeat the tasks.

- Decisions about treatment should be based on the data obtained from the evaluation of the patient.

- Facilitate generalization from the beginning of treatment.

- Be flexible and adapt therapy to each patient (Ríos., et al. 2007).

\section{Results}

\section{Patient 1}

The first patient presented a moderate design syndrome, with altered episodic memory, language (mixed aphasia) and depressive symptomatology without reaching diagnostic criteria of major depressive disorder. The changes observed after cranioplasty together with neuropsychological rehabilitation sessions were as follows:

- In memory tasks there was an improvement in retention percentage ( $75 \%$ compared to $25 \%$ before cranioplasty). 
- In terms of recognition tasks, the patient obtained a scalar score of 3 in the initial evaluation. Tras the surgical intervention improved 3 points and after completing the neuropsychological rehabilitation managed to place himself in a scalar score of 11 .

- There was an improvement in executive functions (go-no go tasks and similarities), keeping phonetic creep slightly altered (18 versus 26 words).

- While in cognitive parameters, the patient presented or a considerable improvement, the level of functionality in their basic activities of daily living remained compromised, requiring some supervision from a family member. The main cognitive sequel was its ability to categorize and abstract.

\section{Patient 2}

He was diagnosed with a dyssejecutive syndrome prior to cranioplasty, with main involvement in working memory, premotor series, go-no-go tasks and conflicting instructions. He had a level of mild depression (score of 13 in BDI) at the beginning of cognitive treatment, with autolytic ideation without structured plan and mild hemiplegia (which forced him to make a lateralization change for writing).

After cranioplasty and together with neuropsychological rehabilitation sessions, changes were observed in: a) working memory, being able to perform 6 correct steps in the TMT-B (cuando at the beginning of treatment was impracticable);b) significant improvement in phonetic creep (words by $\mathrm{P}$ and categorial evocations of animals), going from 12 to 22 words and from 19 to 31, respectively; c) the visuoconstructive praxis measured with the Complex Figure of King were within the normality after surgical and cognitive treatment; and d) at the clinical level, depressive symptomatology and autolytic ideation disappeared (score of 2 in BDI). The patient's functionality was more satisfactory, being able to perform BVBD and instruments autonomously. We cannot say that the patient recovered his premobid level, but he only showed a more satisfactory functional evolution, despite presenting spasticity in the left upper extremity and some difficulty in gait.

\section{Discussion}

After plasty there is a slight acceleration in the recovery of certain cognitive processes, which stabilize and reach their ceiling after a few months. It has been observed that after joint treat- ment (surgical and neuropsychological) there is an increase in the perception of personal self-efficacy. Cranioplasty produces clinical benefits beyond mere protection and aesthetic improvement, in the same line of other studies [17-20] that report an objective improvement of $40 \%$ and subjective improvement of $56 \%$ in these patients. According to these results, it can be said that executive functions improve when neuropsychological rehabilitation sessions are incorporated together with cranioplasty, in addition to decreasing the comorbid depressive symptomatology.

\section{Conclusion}

Neuropsychological rehabilitation is a global treatment that not only includes pencil and paper exercises (brain training), but also needs to include experiences that have an ecological validity and thus achieve transfer of what has been learned to the patient's natural environment. That is why, from the Rehabilitation service of our hospital, we not only include these exercises, but we also work with interfamily groups to promote coping and generate a better overall patient that prepares them for their normal daily life. It is also included, within the therapeutic approach, sessions of systemic family therapy to favor the processes of differentiation after an $\mathrm{AD}$.

Craniopathy is necessary and it is advisable to complement it with neuropsychological rehabilitation. In the two patients we have seen and presented in this study, a rapid evolution was observed after thelastia craniop (an aspect that probably has to do with the increase in cerebral irrigation). Although, after a certain time, certain parameters stabilized and its performance decreased slightly. There are other cognitive aspects that improved considerably.

It is interesting to note that patient 2 , his apparent damage being more severe, his functional recovery was more prominent, requiring less supervision after discharge. This may be because left-handed people could develop skills that correspond to different hemispheres, making them less vulnerable to injuries from a dominant hemisphere, as some authors claim [29-34].

To conclude, we believe that the different health specialties (neurology, neurosurgery, physical medicine and rehabilitation, clinical psychology...), should work in an interdisciplinary way so that patients can be recipients of what research brings from the different disciplines. 


\section{Conflict of Interest}

The authors declare that they have no conflict of interest.

\section{Bibliography}

1. Price B H., et al. "The comportmental learning disabilities of early frontal lobe damage". Brain: A Journal of Neurology 113 (1990): 1383-1393.

2. Pelegrin $\mathrm{C}$ and Tirapu J. "Neuropsychiatry of traumatic prefrontal damage". Monographs of Psychiatry 7 (1995): 11-21.

3. Verdejo-García Antonio and Antoine Bechara. "Neuropsicología de las funciones ejecutivas" [Neuropsychology of executive functions]". Psicothema 22.2 (2010): 227-235.

4. Sohlberg MM and CA Mateer. "Training use of compensatory memory books: a three stage behavioral approach". Journal of Clinical and Experimental Neuropsychology 11.6 (1989): 871891.

5. Kolb Bryan and Ian Q Whishaw. "Fundamentals of human neuropsychology". Macmillan (2009).

6. Read D E. "Solving deductive-reasoning problems after unilateral temporal lobectomy". Brain and Language 12.1 (1981): 116-127.

7. Blumer D and Walker E. "The neural basis of sexual behavior". In D.F. Benson and F. Blumer, eds. Psychiatric Aspects of Neurologic Disease. New York: Grune and Stratton (1975).

8. Blumer D and Benson D. "Personality changes with frontal and temporal lesions". In D.F. Benson and F. Blumer, eds. Psychiatric Aspects of Neurologic Disease. New York: Grune and Stratton (1975).

9. Machado Sergio., et al. "Hemispheric specialization and regulation of motor behavior from the perspective of cognitive neuroscience". Mental Health 36.6 (2013): 513-520.

10. Cooper D James., et al. "Decompressive craniectomy in diffuse traumatic brain injury". The New England Journal of Medicine 364.16 (2011): 1493-1502.

11. Hutchinson Peter J., et al. "Trial of Decompressive Craniectomy for Traumatic Intracranial Hypertension". The New England Journal of Medicine 375.12 (2016): 1119-1130.

12. González de Santiag MA., et al. "Cranial reconstruction with custom implant". Revista Española de Cirugía Oral y Maxilofacial 33.1 (2011): 40-44.

13. Cooker T. "The therapy of intracranial pressure". In Holder A ed: Brain scrounging, intracranial pressure and surgical inter- ventions in brain diseases. Vienna: A Holder (1901): 262-266.

14. Lacerda Gallardo AJ., et al. "Decompressive craniectomy in the management of head trauma. A surgical option". Revista Mexicana de Neurocirugía 5.6 (2004): 564-570.

15. Lacerda Gallardo AJ., et al. "Protocol for early surgical treatment in the control of intracranial hypertension in severe head trauma". Revista Chilena de Neurocirugía 29 (2007): 45-51.

16. Lacerda Gallardo AJ., et al. "Decompressive hemicraniectomy as a treatment of malignant cerebral infarction". Revista Chilena de Neurocirugía 28 (2007): 34-40.

17. Winkler PA., et al. "Influence of cranioplasty on postural blood flow regulation, cerebrovascular reserve capacity, and cerebral glucose metabolism". Journal of Neurosurgery 93 (2000): 53-61.

18. Suzuki N., et al. "Neurological improvement after cranioplasty. Analysis by dynamic CT scan". Acta Neurochirurgica 122.1-2 (1993): 49-53.

19. Won Yoo-Dong et al. "Cranioplasty effect on the cerebral hemodynamics and cardiac function". Acta Neurochirurgica 102 (2008): 15-20.

20. Paredes Igor., et al. "Cranioplasty after decompressive craniectomy. A prospective series analyzing complications and clinical improvement". Neurocirugia (Asturias, Spain) 26.3 (2015): 115-125.

21. Erdogan E., et al. "The effect of cranioplasty on cerebral hemodynamics: evaluation with transcranial Doppler sonography". Neurology India 51.4 (2003): 479-481.

22. Reitan Ralph M. "Validity of the Trail Making Test as an indicator of organic brain damage". Perceptual and Motor Skills 8.3 (1958): 271-276.

23. Wechsler D. "Adaptation to Spanish of the Wechsler-III memory scale". Madrid: TEA Ediciones (2004).

24. Folstein M F., et al. "'Mini-mental state'. A practical method for grading the cognitive state of patients for the clinician". Journal of Psychiatric Research 12.3 (1975): 189-198.

25. Peña-Casanova J. "Normality, semiology and neuropsychological pathology. Integrated Neuropsychological Exploration Program. Test Barcelona Revised (2nd ed.)”. Barcelona: Masson (2004).

26. King A. "Copy Test of a complex figure”. Manual. Spanish adaptation. Madrid: TEA EDICIONES (1997).

27. Dubois B., et al. "The FAB: a Frontal Assessment Battery at bedside". Neurology 55.11 (2000): 1621-1626. 
28. Sanz, Jesús., et al. "Spanish Adaptation of the Inventory for Beck-II Depression (BDI-II): 3. Psychometric Properties in Patients with Psychological Disorders". Clinic and Health 16.2 (2005): 121-142.

29. Zuliani L. "The use of both hands enables further development". Revista Médica Pediátrica. Official Organ of the Colombian Society of Pediatrics 38.2 (2003).

30. Ríos-Lago M., et al. "Alteraciones de la atención tras daño cerebral traumático: evaluación y rehabilitación". Revista de Neurología 44.5 (2007): 291-297.

31. Sohlberg MM and Mateer CA. "Cognitive rehabilitation: an integrative neuropsychological approach”. 1 ed. New York: Guilford Press (2001).

32. Espinosa-Gil Rosa María and Monteagudo Santamaría María. "Inter-Family/Intercultural Group/and Acquired Brain Injury". EC Psychology and Psychiatry 9.10 (2020): 09-18.

33. Baddeley A., et al. "Testing central executive with a pencil and paper test. In Rabbitt P. "Methodology of frontal and executive function”. Hove, UK: Psychology Press (1997).

34. Muñoz JM and Tirapu J. "Rehabilitación de las funciones ejecutivas”. Revista de Neurología 38.7 (2004): 656-663.

\section{Volume 4 Issue 8 August 2021}

(C) All rights are reserved by Espinosa-Gil Rosa María.,

et al. 